\title{
Anatomia foliar e radicular comparativa de sete espécies de Catasetum Rich. ex Kunth (Orchidaceae: Catasetinae)
}

\author{
Comparative leaf and root anatomy of seven species of Catasetum Rich. ex Kunth \\ (Orchidaceae: Catasetinae)
}

\author{
M. S. Oliveira ${ }^{1 *}$; A. W. C. Ferreira ${ }^{2}$; H. C. Oliveira ${ }^{3}$ \\ ${ }^{1}$ Programa de Pós-Graduação em Biodiversidade, Ambiente e Saúde, Universidade Estadual do Maranhão, 65604- \\ 090, Praça Duque de Caxias, s/n, Morro do Alecrim, Caxias-MA, Brasil. \\ ${ }_{2}^{2}$ Departamento de Biologia, Universidade Federal do Maranhão, CEP 65080-805, Cidade Universitária Dom \\ Delgado, Av. dos Portugueses, 1966, Bacanga, São Luís-MA, Brasil. \\ ${ }_{3}^{3}$ Programa de Pós-Graduação em Biodiversidade, Ambiente e Saúde, Universidade Estadual do Piauí, Campus \\ Heróis do Jenipapo, 64280-000, Av. Sto. Antônio s/n, Bairro São Luís, Campo Maior-PI, Brasil. \\ *miguelsena2010@hotmail.com \\ (Recebido em 24 de março de 2021; aceito em 11 de dezembro de 2021)
}

O gênero Catasetum é um grupo complexo e pouco se conhece sobre a composição anatômica de suas folhas e raízes. O presente estudo objetivou descrever e comparar anatomicamente a estrutura foliar e radicular de sete espécies e contribuir com o conhecimento anatômico e taxonômico das espécies incluídas na subtribo Catasetinae. As amostras foram fixadas em FAA70 e preservadas em etanol 70\%. As secções foram realizadas a mão livre, clarificadas em hipoclorito de sódio $50 \%$ e, posteriormente, coradas com fucsina básica e azul de Astra $0,05 \%$. As lâminas foram montadas com gelatina glicerinada e lutadas com esmalte incolor. Os espécimes estudados são estruturalmente semelhantes entre si, com folhas de epiderme uniestratificada, cutícula delgada e lisa em ambas as faces, colênquima angular, esclerênquima circundando o feixe vascular da nervura principal e mesofilo homogêneo e raízes com epivelame distinguível, velame multiestratificado com espessamento secundário, exoderme uniestratificada com presença de tilossomos, córtex parenquimático multiestratificado, endoderme e periciclo uniestratificado e cilindro vascular poliarco, estando esses dados de acordo com a literatura para o gênero. $O$ registro de folhas anfiestomaticas são aqui descritas pela primeira vez para a anatomia das Catasetum. Contudo, não foram encontrados marcadores anatômicos úteis para diferenciar as espécies estudas ou que contribuíssem para fins taxonômicos ou com a sistemática deste gênero.

Palavras-chave: estudos anatômicos, epífitas, orquídea.

The genus Catasetum is a complex group, and little is known about the anatomical composition of its leaves and roots. The present study aimed to describe and anatomically compare the leaf and root structure of seven species and contribute to the anatomical and taxonomic knowledge of the species included in the Catasetinae subtribe. The samples were fixed in FAA70 and preserved in 70\% ethanol. The sections were performed freehand, clarified in 50\% sodium hypochlorite, and later stained with basic fuchsin and $0.05 \%$ Astra blue. The slides were mounted with glycerin gelatin and matched with colorless enamel. The specimens studied are structurally similar to each other, with unistratified epidermal sheets, thin and smooth cuticle on both sides, angular collenchyma, sclerenchyma surrounding the vascular bundle of the main vein and homogeneous mesophyll and roots with distinguishable epivelamis, multistratified canopy with secondary thickening, unistratified exoderm with the presence of tylossomes, multistratified parenchymal cortex, unistratified endoderm and pericycle, and polyarc vascular cylinder. These data are found in accordance with the literature for the genus. Records of amphistomatic leaves are described here for the first time for the anatomy of Catasetum. However, no useful anatomical markers were found to differentiate the species studied or that would contribute to taxonomic purposes or to the systematics of this genus.

Keywords: anatomical studies, epiphytic, orchid.

\section{INTRODUÇÃO}

Orchidaceae Juss., com 736 gêneros e cerca de 28.000 espécies, é umas das maiores famílias botânicas entre as angiospermas, e suas espécies estão amplamente distribuídas em quase todo o planeta, não sendo encontradas apenas em desertos e nos pólos [1-3], amplamente diversificada em ambientes tropicais e subtropicais [4]. Atualmente, a família encontra-se subdividida em cinco 
subfamílias, são elas: Apostasioideae, Cypripedioideae, Vanilloideae, Orchidoideae e Epidendroideae $[1,5]$.

A subtribo Catasetinae (Epidendroideae) compreende oito gêneros [6-8] e dentre estes, destaca-se Catasetum Rich. ex Kunth que, com 130 espécies, é considerado o maior da subtribo, ocorrendo desde o México até o norte da Argentina $[9,10]$. No Brasil, estão registradas cerca de 114 espécies, sendo que 14 delas ocorrem no estado do Maranhão [11].

As espécies de Catasetum são predominantemente epífitas [12, 13], porém podem ser encontradas ocasionalmente como terrestres ou rupícolas [14]. O sucesso ecológico que as espécies epífitas possuem está relacionado com algumas adaptações morfológicas e funcionais [15], tais como raízes com epiderme multiestratificada e velame capaz de absorver água e sais minerais, oferecer proteção mecânica e reduzir a transpiração [16]. Tais adaptações devem ser analisadas afim de testar sua utilização como marcadores anatômicos.

O gênero Catasetum possui espécies que apresentam características morfológicas vegetativas semelhantes, sendo as flores estaminadas o principal critério utilizado para diferenciar as espécies pois, ao contrário das flores pistiladas, apresentam variações morfológicas significativas [17].

Devido às semelhanças morfológicas na parte vegetativa e nas flores pistiladas das espécies de Catasetum, os estudos anatômicos podem incluir outras informações além das fornecidas pelas flores estaminadas temporárias e efêmeras. Por isso, trabalhos morfoanatômicos para fins taxonômicos, ecológicos e fisiológicos têm sido desenvolvidos com diversas partes vegetativas de espécies de Catasetum (folhas, caules, raízes) [18, 19].

No Brasil, poucos são os trabalhos realizados com anatomia de Catasetinae, podendo ser mencionados os de Stern e Judd (2001) [20] com a anatomia dos órgãos vegetativos de Catasetinae da região amazônica, o de Pedroso-de-Moraes et al. (2012) [21] que descreveu a estrutura radicular de doze espécies nativas brasileiras e o de Silva et al. (2015) [22] com o uso de marcadores anatômicos radiculares de oito espécies de Catasetum da Região do Portal da Amazônia, no estado do Mato Grosso. Entretanto, ainda são escassas as informações sobre a organização foliar e radicular de diversas espécies de Catasetum.

O presente estudo teve como objetivo investigar a anatomia foliar e radicular de sete espécies de Catasetum e alguns de seus morfotipos florais, visando identificar estruturas que possam diferenciá-los e contribuir com o conhecimento taxonômico e anatômico de Catasetum e da subtribo Catasetinae.

\section{MATERIAL E MÉTODOS}

\subsection{Espécimes utilizados}

Parte do material utilizado neste estudo (Figura 1) foi coletado por Oliveira et al. (2021) [23], em áreas do Domínio da Amazônia e do Cerrado, em quatro municípios da região central do Leste

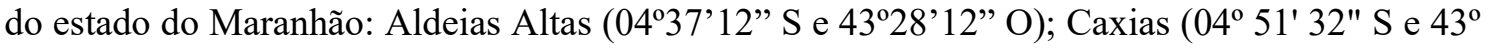
21' 22" O); Codó $\left(04^{\circ} 27^{\prime} 12,8^{\prime \prime}\right.$ S e $43^{\circ} 53^{\prime} 01,7^{\prime \prime}$ O) e São João do Sóter $\left(05^{\circ} 06^{\prime} 36^{\prime \prime}\right.$ S e $43^{\circ} 48^{\prime} 36^{\prime \prime}$ O) [24-28]. 


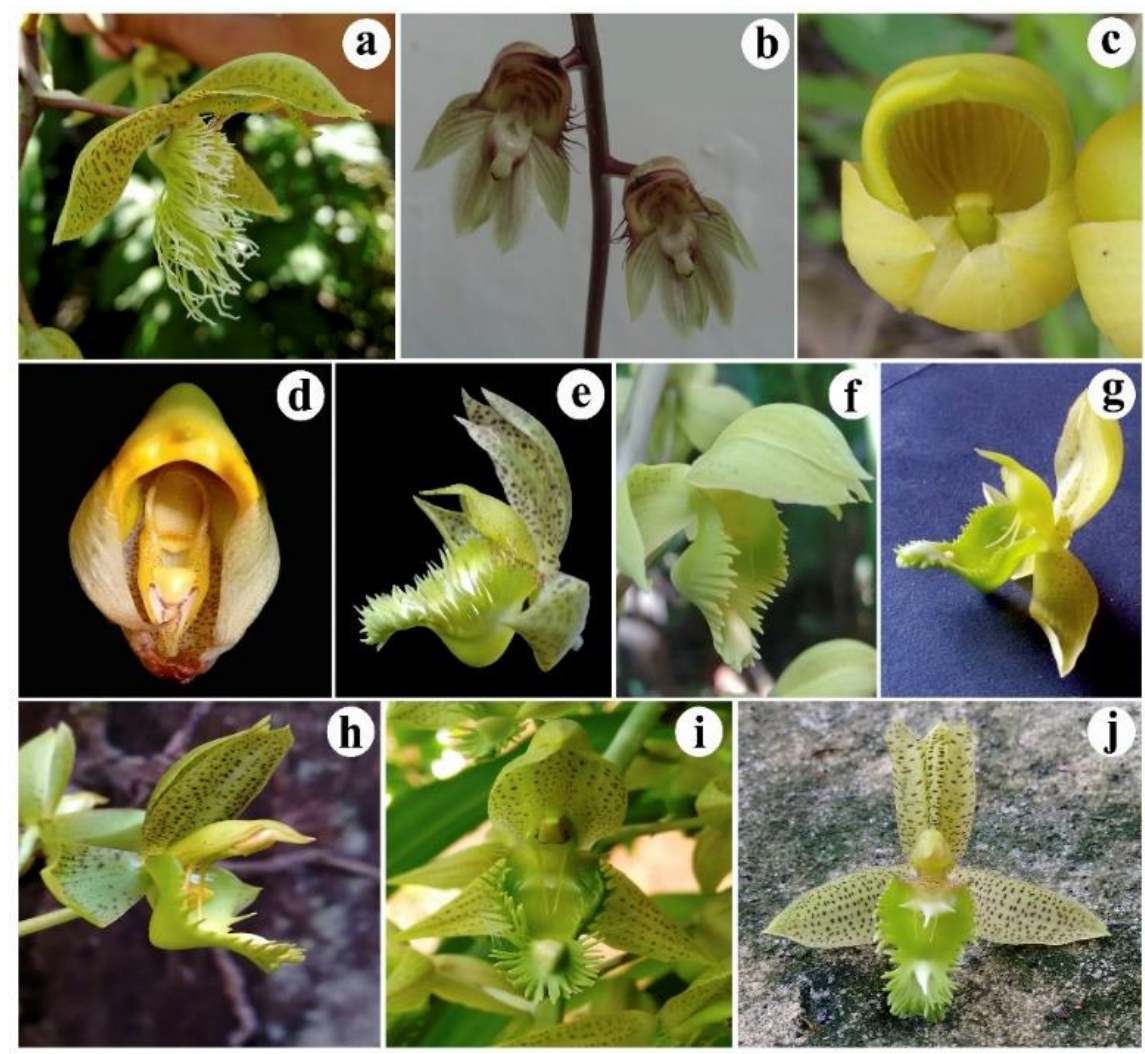

Figura 1. Flores das espécies de Catasetum coletadas na região central do Leste Maranhense: $a$. $\underline{C}$.

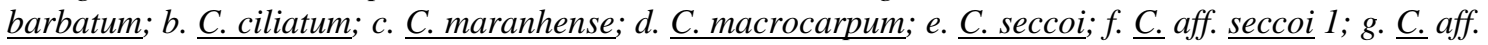

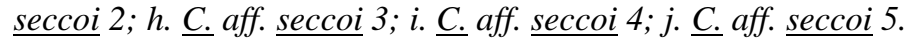

Neste estudo foram utilizadas sete espécies e cinco morfotipos. As espécies coletadas por Oliveira et al. (2021) [23] foram Catasetum barbatum (Lindl.) Lindl. (três indivíduos), C. ciliatum Barb. Rodr. (um indivíduo), C. maranhense K.G. Lacerda \& J.B.F. Silva (dois indivíduos), $C$. macrocarpum Rich.ex Kunth (dois indivíduos), C. seccoi M.F.F. Silva \& A.T. Oliveira (um indivíduo) e seus cinco morfotipos florais (cinco indivíduos) (Figura 1). As espécies C. discolor (Lindl.) Lindl (um indivíduo) e C. osculatum K.G. Lacerda \& V.P. Castro (um indivíduo) foram obtidas através de doação por um orquidófilo de seu cultivo particular (Tabela 1).

Tabela 1. Espécies, quantidade de indivíduos e órgãos analisados. Nota: Quantidade de Indivíduos (Q.I); presença (x), ausência (-); Ep: epífita.

\begin{tabular}{|c|c|c|c|c|c|}
\hline Espécies & Hábito & Q.I. & Folhas & Raízes & Vouchers \\
\hline \multirow{3}{*}{ Catasetum barbatum (Lindl.) Lindl. } & Ep & 3 & $\mathrm{x}$ & $\mathrm{x}$ & HABIT 3428 \\
\hline & & & & & HABIT 3705 \\
\hline & & & & & HABIT 3430 \\
\hline \multirow{2}{*}{ Catasetum macrocarpum Rich. ex Kunth } & Ep & 2 & $\mathrm{x}$ & $\mathrm{x}$ & HABIT 3431 \\
\hline & & & & & HABIT 3432 \\
\hline \multirow{2}{*}{ Catasetum maranhense K.G. Lacerda \& J.B.F. Silva } & Ep & 2 & $\mathrm{x}$ & $\mathrm{x}$ & HABIT 3423 \\
\hline & & & & & HABIT 3424 \\
\hline Catasetum ciliatum Barb. Rodr. & Ep & 1 & $\mathrm{x}$ & $\mathrm{x}$ & MAR 11.513 \\
\hline Cataseum discolor (Lindl.) Lindl. & Ep & 1 & - & $\mathrm{x}$ & In vivo \\
\hline Catasetum osculatum K.G. Lacerda \& V.P. Castro & Ep & 1 & - & $\mathrm{x}$ & In vivo \\
\hline Catasetum seccoi M.F.F. Silva \& A.T. Oliveira & Ep & 1 & $\mathrm{x}$ & $\mathrm{x}$ & HABIT 3419 \\
\hline Catasetum aff. seccoi 1 & Ep & 1 & $\mathrm{x}$ & $\mathrm{x}$ & HABIT 3561 \\
\hline Catasetum aff. seccoi 2 & Ep & 1 & $\mathrm{x}$ & $\mathrm{x}$ & HABIT 3564 \\
\hline Catasetum aff. seccoi 3 & Ep & 1 & $\mathrm{x}$ & $\mathrm{x}$ & HABIT 3565 \\
\hline Catasetum aff. seccoi 4 & Ep & 1 & $\mathrm{x}$ & $\mathrm{x}$ & HABIT 3563 \\
\hline Catasetum aff. seccoi 5 & Ep & 1 & $\mathrm{x}$ & $\mathrm{x}$ & HABIT 3562 \\
\hline
\end{tabular}




\subsection{Procedimentos Metodológicos}

Para as análises anatômicas das raízes e folhas, retirou-se de cada indivíduo três amostras (ca. $3 \mathrm{~cm}$ ) das folhas adultas (ápice, meio, base e margem) e três amostras de raízes jovens (ápice, meio e base), sendo seus respectivos vouchers incorporados aos acervos dos herbários HABIT e MAR [29] (Tabela 1).

As amostras foram fixadas em FAA70 (Formaldeído, Ácido acético glacial e Álcool etílico $70 \%$ ) por 48 horas e preservadas em etanol 70\% [30]. As secções transversais foram realizadas a mão livre, clarificados em hipoclorito de sódio a $50 \%$ (solução comercial), corados com fucsina básica e azul de Astra 0,05\% [31]. Posteriormente, as lâminas foram montadas com gelatina glicerinada e lutadas com esmalte incolor [32]. O diâmetro das raízes (45 no total) foi obtido com a utilização de um paquímetro Universal 150mm (Digimess).

A pesquisa foi realizada no Laboratório de Anatomia Vegetal (LABIO) da Universidade Estadual do Piauí, Campus Heróis do Jenipapo em Campo Maior-PI e no Laboratório de Ciências Biológicas (LCB) da Universidade Estadual do Maranhão (CESC/UEMA). As fotomicrografias foram obtidas através de capturador de imagem acoplado ao microscópio Oleman (N-180m) com auxílio do software BelCapture v.1.1 e, posteriormente, editadas no software Photoshop ${ }^{\circledR}$ CS5 v. 12.0 (Adobe ${ }^{\circledR}$ Systems).

As espécies Catasetum osculatum K.G. Lacerda \& V.P. Castro e Catasetum discolor (Lindl.) Lindl. tiveram apenas as raízes analisadas, estando as folhas indisponíveis no período correspondente a realização da pesquisa.

\section{RESULTADOS E DISCUSSÃO}

Foram analisados 16 indivíduos de sete espécies e cinco morfotipos de Catasetum. Nas análises de folhas, constatou-se que todas as regiões (ápice, meio e base) das sete espécies compartilhavam características como epiderme uniestratificada, cutícula delgada e lisa em ambas as faces, colênquima angular no mesofilo, esclerênquima circundando o feixe vascular da nervura principal e mesofilo homogêneo (Figura 2a).

A cutícula delgada e lisa foi observada em ambas as faces de todas as amostras das sete espécies e dos morfotipos florais de Catasetum (Figuras 2a-h). Esses resultados combinam com outros trabalhos com espécies desse gênero e de outras Orchidaceae [33, 34]. De acordo com Zanenga-Godoy e Costa (2003) [35], a exposição ao sol é o principal fator que determina o padrão de espessamento da cutícula e, quanto maior for a exposição, mais as folhas tenderão a exibir uma cutícula. As espécies amostradas neste estudo foram coletadas em locais sombreados ou com pouca incidência de luz e, por isso, possuíam cutícula delgada, estando assim os dados encontrados de acordo com a literatura para o gênero.

A presença de tricomas não foi constatada em nenhuma das espécies analisadas. Atualmente, ainda não existem informações na literatura sobre a presença, ausência ou distribuição de tricomas em folhas de Catasetum. É conhecido para o gênero apenas o registro de tricomas feito por Franken et al. (2016) [17] nos osmóforos das espécies da aliança Catasetum cristatum.

As folhas das espécies estudadas são predominantemente hipoestomáticas (Figuras 2b-c), apresentando estômatos situados no mesmo nível que as demais células epidérmicas ordinárias, com projeções cuticulares em forma de crista e câmaras subestomáticas bem desenvolvidas (Figura 2c). No entanto, somente nas amostras de ápice foliar de Catasetum aff. seccoi 2 (Figura 2d) e Catasetum aff. seccoi 5 (Figura 2e), foram constatadas a presença de estômatos em ambas as faces (anfiestomáticas), sendo esse registro inédito para espécies do gênero Catasetum.

Exceto pelo que foi verificado nas duas amostras do ápice foliar de $C$. aff. seccoi 2 e 5, nossos resultados estão de acordo com os estudos sobre a anatomia foliar com plantas do Cerrado, que mostram que a maioria delas apresenta folhas hipoestomáticas $[18,36,37]$ e que a presença de câmaras subestomáticas é uma característica comum em orquídeas epífitas adaptadas a elevadas temperaturas e a pouca disponibilidade de água $[38,39]$.

A epiderme é uniestratificada, constituída por células epidérmicas adaxiais maiores que as abaxiais (Figuras 2e-g), sendo essa uma característica, recorrente para Catasetum [33, 34, 39], podendo estar relacionada com a função de estocagem de água ou estratégia de proteção contra a alta e excessiva intensidade solar [40, 41]. 


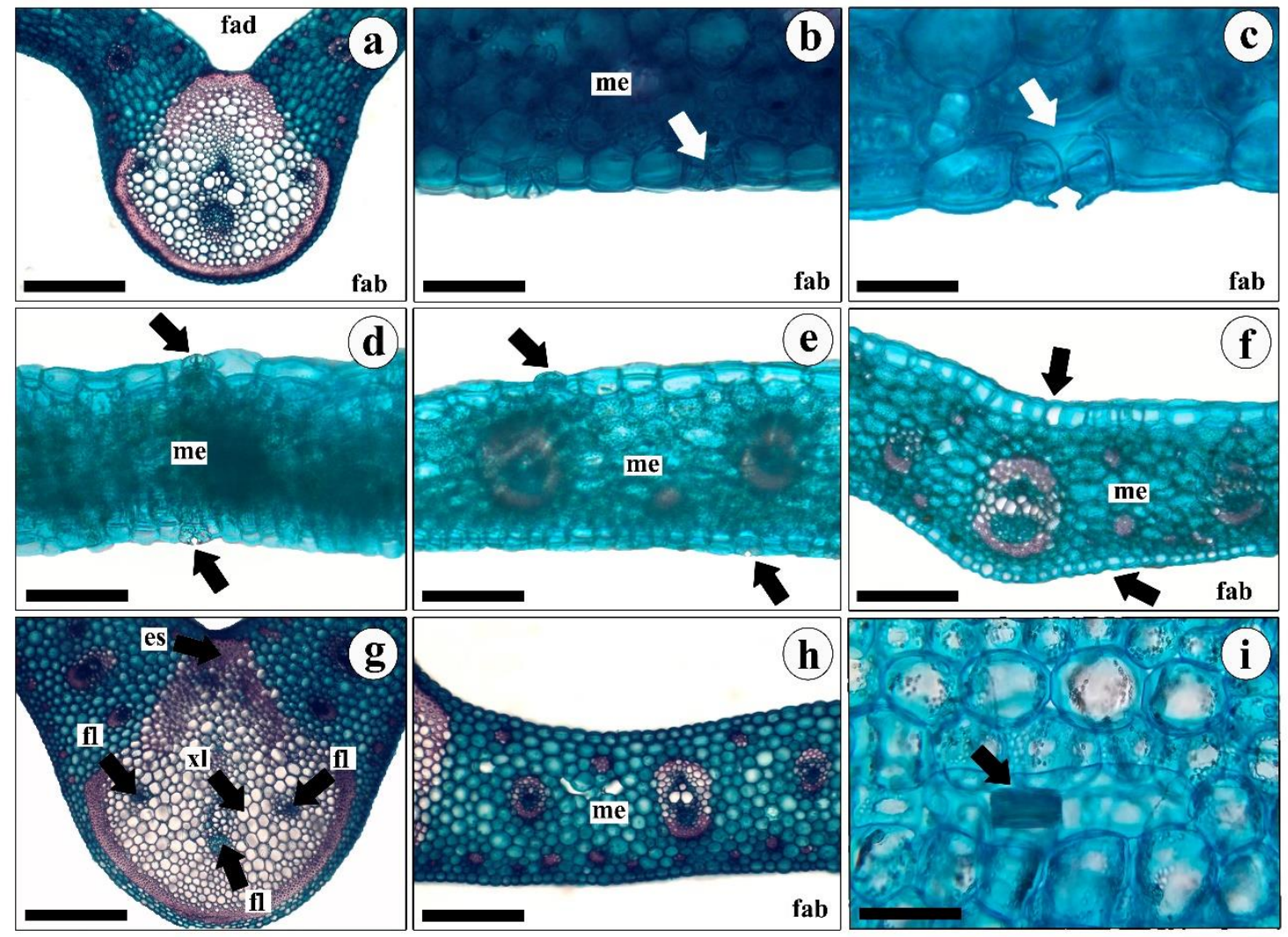

Figura 2. Sessões foliares das espécies estudadas: a. Visão geral da nervura principal da região mediana

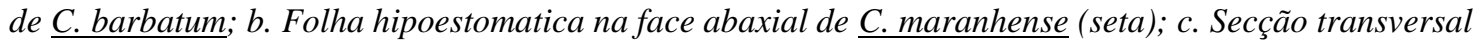

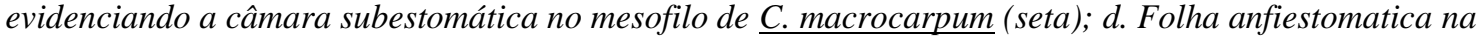
região apical de Catasetum aff. seccoi 2 (seta); e. Folha anfiestomatica na região apical de Catasetum aff. seccoi 5 (seta); f. Células epidérmicas adaxiais maiores que as abaxiais na região apical de $\underline{C}$. ciliatum; $g$. Secção transversal evidenciando o feixe vascular colateral e periféricos anfivasais na região

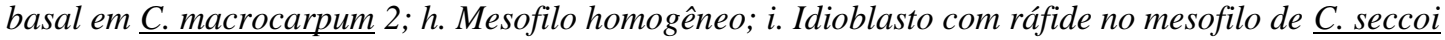

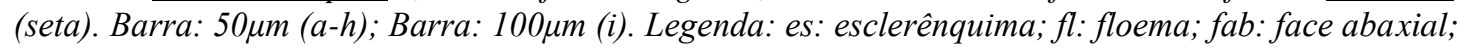
fad: face adaxial; me: mesofilo; xl: xilema.

O mesofilo de todas as amostras analisadas apresentou-se homogêneo (Figuras 2h-i), constituído de parênquima clorofiliano regular, caracteristicamente observado na maioria das espécies de Orchidaceae [33, 42, 43] com presença de conteúdo intracelular e de idioblastos com ráfides (Figura 2i).

Idioblastos com ráfides foram visualizados distribuídos aleatoriamente em diversas partes do mesofilo, sendo a presença dos mesmos comum em Orchidaceae [34], e podem estar relacionados tanto a processos químicos de balanceamento iônico, osmoregulação ou como adaptativos ao herbivorísmo, tornando-as menos palatáveis aos animais [44, 45].

A nervura foliar principal de todas as espécies apresentou contorno côncavo convexo (sentido adaxial-abaxial) e feixe vascular com conformação plano convexo. O sistema vascular apresentou organização variável de acordo com a região analisada (ápice, meio, base). As amostras de ápice possuem organização exclusivamente colaterais, enquanto que as regiões do meio e base são colaterais com feixes periféricos anfivasais (Figuras 2a, 2f, 2g).

Nas análises das raízes, constatou-se que as espécies possuem características comuns: epivelame distinguível, velame multiestratificado com espessamento secundário, exoderme uniestratificada com presença de tilossomos, córtex parenquimático multiestratificado, endoderme e periciclo uniestratificados e cilindro vascular poliarco (Figura 3a). Essas estruturas são conhecidas em estudos anteriores para o gênero Catasetum [22] e compartilhadas com outras monocotiledôneas [46-48].

Os pêlos radiculares são unicelulares e ocorreram nas três regiões das raízes amostradas (ápice, meio e base) dos 14 espécimes (Figura 3b), com exceção de $C$. discolor e $C$. osculatum, que não 
apresentaram pelos em nenhuma das três regiões. Os pelos radiculares têm a função de proteger, fixar e ampliar a absorção de água e nutrientes a partir de um substrato ou do ar [48, 49] e a presença destes foram também verificadas em outros trabalhos com orquídeas epífitas e terrestres $[50,22]$ e em outras famílias botânicas [51, 52].
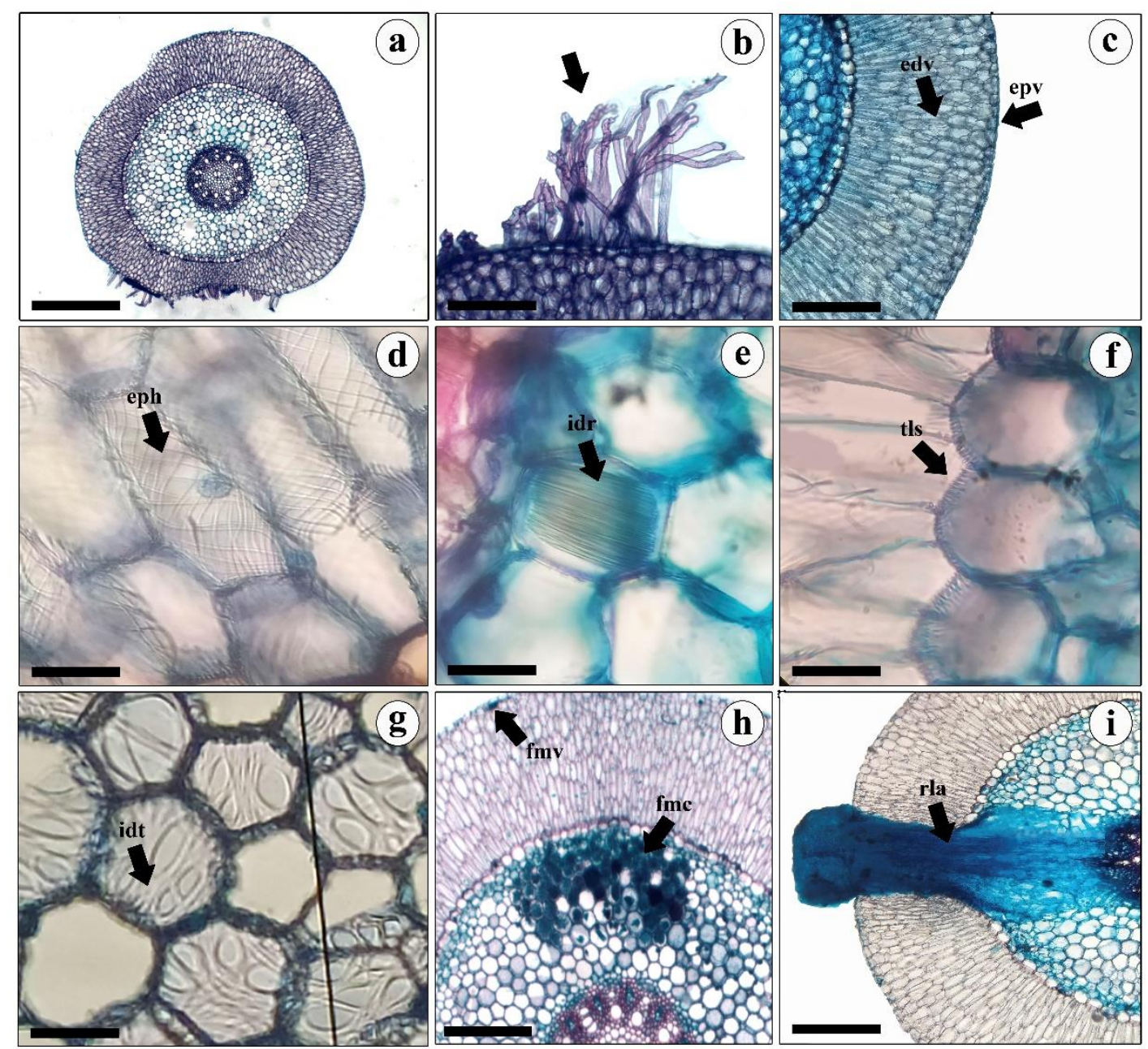

Figura 3: Sessões radiculares das espécies estudadas- $a$. Visão geral da Raiz em secção transversal; $b$. Pêlos radiculares; c. Diferenciação do Velame em epivelame e endovelame; $d$. Espessamentos de parede nas células do velame (seta); e. Idioblasto com Ráfides (seta); f. Tilossomos (seta); g. Idioblastos traqueioidais (seta); $h$. Fungos micorrizos no velame e córtex (seta); $i$. Crescimento de raiz lateral. Barra: $50 \mu m$ (a,b,c,h,i); Barra: $100 \mu m$ (d,e,f,g). Legenda: epv: epivalame; edv: endovelame; eph: espessamento helicoidais; idr: idioblasto com ráfides; tls: tilossomos; Idioblastos traqueioidais; fmv: fungos micorrizos no velame; fmv: fungos micorrizos no cortex; rla: raiz lateral.

O velame apresentou-se diferenciado em duas camadas distintas, uma externa uniestratificada (epivelame) constituída por células de parede celulósica e outra interna multiestratificada (endovelame), presentes em todas as espécies analisadas neste estudo (Figura 3c) e em outras Catasetum [22, 53]. O número de camadas do velame variou de acordo com a espécie e a região analisada (Tabela 2) e suas células possuem espessamento secundário dispostos em faixas helicoidais (Figura 3d) que podem ser celulósicas ou lignificadas [20, 21].

O velame é um tecido vegetal presente não só nas raízes das Orchidaceae, mas também em outras famílias botânicas (Araceae, Bromeliaceae, Liliaceae, Dioscoreaceae, Taccaceae, Amaryllidaceae e Commelinaceae), sendo responsável pela proteção mecânica do interior da raiz, trocas gasosas e absorção da umidade do ar, sais minerais e nutrientes oriundos de substâncias líquidas orgânicas [33, 52, 54-58]. Portanto, o velame é imprescindível para processos físicos, químicos e fisiológicos dessas espécies. 
A exoderme em todas as espécies é uniestratificada, constituída de células espessadas em forma de "U", com presença de idioblastos com ráfides (Figura 3e) e tilossomos (Figura 3f). O córtex de todas as espécies é multiestratificado e parenquimático, constituído de células isodiamétricas, com número de camadas que variam desde a espécie a região analisada (Tabela 2), com células apresentando fibras em forma de faixas (Figura 3g). Foi verificada a presença de fungos micorrizos e idioblastos com ráfides (Figuras $3 \mathrm{~h}$-i).

A endoderme radicular é uniestratificada com paredes espessadas predominantemente em forma de "O" e foi observada em todos os espécimes estudados (Figuras 3a, h) e em outras espécies de Catasetinae [20,21]. O cilindro vascular de todas as espécies tem estrutura poliarca, formado por cordões alternados de floema e xilema (Figuras 4a-1), e um periciclo uniestratificado que, em algumas amostras, foi observado o crescimento de raiz lateral (Figura 3i).

No cilindro vascular radicular dos indivíduos, o número e a ornamentação dos polos de protoxilema variou da espécie e até mesmo em diferentes regiões da mesma raiz (Figuras 4a-l), como também observado por Silva et al. (2015) [22] e Silva et al. (2010) [50]. A medula das raízes é constituída de parênquima, com células isodiamétricas de paredes finas ou espessas (Figuras 4a-1), como também verificado em outros estudos [21, 50]. 


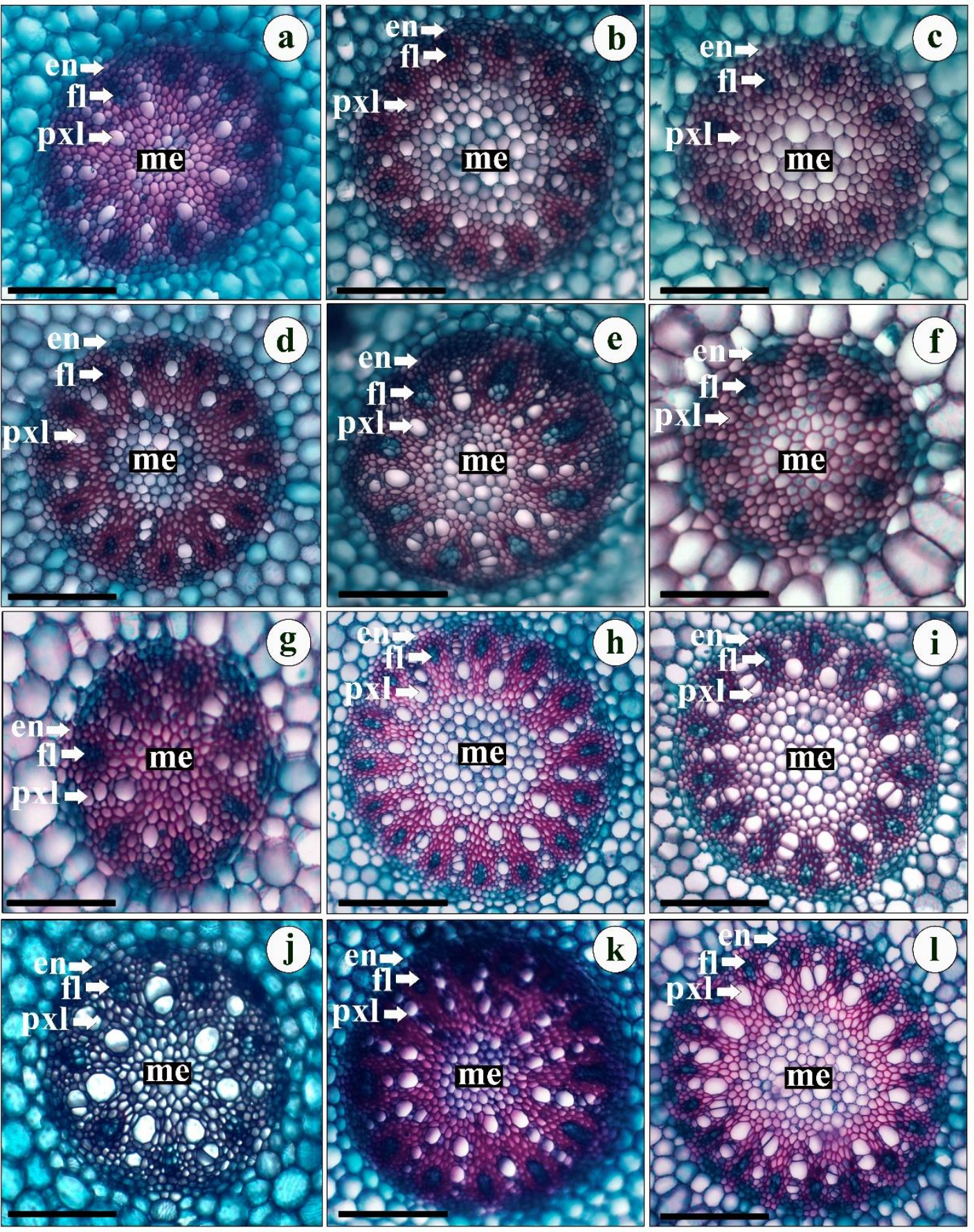

Figura 4. Secções transversais da região mediana de raízes de espécies de Catasetum, evidenciando a ornamentação do cilindro vascular e as variações no número de polos de protoxilema. $a$. C. barbatum, $b$.

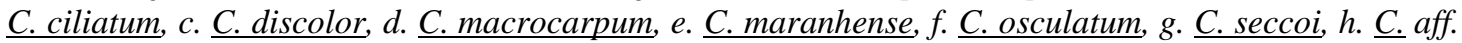

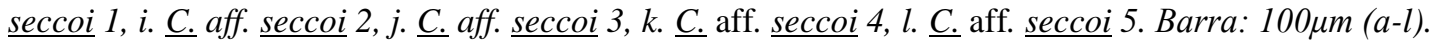
Legendas: en: endoderme; fl: floema; me: medula; pxl: protoxilema.

Nas raízes dos indivíduos analisados (Tabela 2) não foi constatada a relação entre a espessura (diâmetro) da raiz e número de polos de protoxilema. As amostras da base das raízes dos espécimes Catasetum aff. seccoi 1 e Catasetum aff. seccoi 3, ambas com 4 mm de diâmetro, foram as mais espessas. No entanto, a espécie Catasetum aff. seccoi 1 possui 20 polos de protoxilema enquanto que Catasetum aff. seccoi 3 possui apenas 14 polos, sendo este número inferior ao encontrado na mesma região (base) em outros espécimes como Catasetum ciliatum (16 polos), Catasetum maranhense 1 (16 polos), Catasetum seccoi (16 polos), Catasetum aff. seccoi 2 (15 polos), Catasetum aff. seccoi 4 (15 polos) e Catasetum aff. seccoi 5 (17 polos), que possuem apenas $3 \mathrm{~mm}$ de diâmetro. 
Tabela 2. Espécies e características analisadas nas raízes de Catasetum. Legenda: $R A=$ Região Analisada; $N C V=$ Número de Camada do Velame; NCC = Número de Camada do Córtex $;$ NPP= Número de Polos de Protoxilema; DMT=Diâmetro; $I D B=$ Idioblastos; $R f=$ Ráfide.

\begin{tabular}{|c|c|c|c|c|c|c|}
\hline Espécies & $\mathbf{R A}$ & NCV & $\mathrm{NCC}$ & NPP & DMT(mm) & IDB \\
\hline \multirow{3}{*}{ Catasetum barbatum 1} & Ápice & $6-8$ & $7-8$ & $7-9$ & $2-3$ & - \\
\hline & Meio & $7-11$ & $7-8$ & $7-8$ & 3 & $\mathrm{Rf}$ \\
\hline & Base & $7-8$ & 8 & $13-14$ & 3 & $\mathrm{Rf}$ \\
\hline \multirow{3}{*}{ Catasetum barbatum 2} & Ápice & $6-7$ & $6-8$ & $11-12$ & $2-3$ & $\mathrm{Rf}$ \\
\hline & Meio & 6 & $7-9$ & 11 & 3 & $\mathrm{Rf}$ \\
\hline & Base & $5-6$ & $7-8$ & 7 & 3 & $\mathrm{Rf}$ \\
\hline \multirow{3}{*}{ Catasetum barbatum 3} & Ápice & $7-8$ & $7-8$ & 11 & 3 & $\mathrm{Rf}$ \\
\hline & Meio & $7-8$ & 8 & 9 & 3 & $\mathrm{Rf}$ \\
\hline & Base & $6-7$ & 8 & 9 & 3 & $\mathrm{Rf}$ \\
\hline \multirow{3}{*}{ Catasetum ciliatum } & Ápice & $4-6$ & $6-10$ & 15 & 3 & $\mathrm{Rf}$ \\
\hline & Meio & 5 & $9-11$ & 12 & 3 & $\mathrm{Rf}$ \\
\hline & Base & $6-10$ & $8-10$ & $12-16$ & 3 & $\mathrm{Rf}$ \\
\hline \multirow{3}{*}{ Catasetum discolor } & Ápice & $4-5$ & $6-9$ & 9 & 2 & $\mathrm{Rf}$ \\
\hline & Meio & $6-7$ & $7-9$ & 9 & 3 & $\mathrm{Rf}$ \\
\hline & Base & $5-6$ & $7-9$ & 9 & 2 & $\mathrm{Rf}$ \\
\hline \multirow{3}{*}{ Catasetum maranhense 1} & Ápice & $4-7$ & $8-11$ & $11-13$ & $2-3$ & $\mathrm{Rf}$ \\
\hline & Meio & 4 & $8-10$ & 8 & 3 & $\mathrm{Rf}$ \\
\hline & Base & $6-7$ & 8 & 16 & 3 & $\mathrm{Rf}$ \\
\hline \multirow{3}{*}{ Catasetum maranhense 2} & Ápice & $3-6$ & $7-9$ & 11 & 2 & - \\
\hline & Meio & 4 & $7-9$ & $6-12$ & 3 & $\mathrm{Rf}$ \\
\hline & Base & $4-5$ & 7 & 12 & 3 & $\mathrm{Rf}$ \\
\hline \multirow{3}{*}{ Catasetum macrocarpum 1} & Ápice & $4-6$ & $4-8$ & $7-9$ & 2 & $\mathrm{Rf}$ \\
\hline & Meio & $6-7$ & $7-9$ & 12 & 3 & $\mathrm{Rf}$ \\
\hline & Base & $6-9$ & $7-8$ & 12 & 3 & $\mathrm{Rf}$ \\
\hline \multirow{3}{*}{ Catasetum macrocarpum 2} & Ápice & $3-6$ & $6-8$ & 6 & $1-2$ & $\mathrm{Rf}$ \\
\hline & Meio & $4-5$ & $8-9$ & $6-7$ & 2 & $\mathrm{Rf}$ \\
\hline & Base & $4-5$ & $7-8$ & 6 & $2-3$ & $\mathrm{Rf}$ \\
\hline \multirow{3}{*}{ Catasetum seccoi } & Ápice & $4-5$ & $7-8$ & $7-8$ & 2 & $\mathrm{Rf}$ \\
\hline & Meio & $3-5$ & 7 & 7 & $2-3$ & $\mathrm{Rf}$ \\
\hline & Base & $6-8$ & $9-10$ & 15 & 3 & $\mathrm{Rf}$ \\
\hline \multirow{3}{*}{ Catasetum osculatum } & Ápice & $3-5$ & $5-6$ & 7 & $1-2$ & $\mathrm{Rf}$ \\
\hline & Meio & 3 & $4-5$ & $6-7$ & $1-2$ & $\mathrm{Rf}$ \\
\hline & Base & 3 & $5-6$ & $6-7$ & $1-2$ & $\mathrm{Rf}$ \\
\hline \multirow{3}{*}{ Catasetum aff. seccoi 1} & Ápice & $4-5$ & $8-10$ & $9-10$ & 2 & $\mathrm{Rf}$ \\
\hline & Meio & $5-6$ & $12-13$ & 15 & 3 & $\mathrm{Rf}$ \\
\hline & Base & $8-10$ & $10-11$ & 20 & 4 & $\mathrm{Rf}$ \\
\hline \multirow{3}{*}{ Catasetum aff. seccoi 2} & Ápice & $5-7$ & $8-9$ & $11-13$ & 2 & - \\
\hline & Meio & $6-8$ & $9-10$ & 13 & 3 & Rf \\
\hline & Base & $6-8$ & $6-9$ & 15 & 3 & $\mathrm{Rf}$ \\
\hline \multirow{3}{*}{ Catasetum aff. seccoi 3} & Ápice & $6-7$ & $7-9$ & $7-11$ & $2-3$ & - \\
\hline & Meio & $6-9$ & $8-9$ & $7-10$ & 3 & $\mathrm{Rf}$ \\
\hline & Base & $7-8$ & $8-10$ & 14 & 4 & $\mathrm{Rf}$ \\
\hline \multirow{3}{*}{ Catasetum aff. seccoi 4} & Ápice & $4-5$ & $7-9$ & 8 & 2 & $\mathrm{Rf}$ \\
\hline & Meio & $4-6$ & $8-11$ & $12-14$ & 3 & Rf \\
\hline & Base & $6-7$ & $8-11$ & $14-15$ & 3 & $\mathrm{Rf}$ \\
\hline \multirow{3}{*}{ Catasetum aff. seccoi 5} & Ápice & $4-5$ & 7 & 8 & 1 & $\mathrm{Rf}$ \\
\hline & Meio & 7 & $8-9$ & $16-17$ & 3 & Rf \\
\hline & Base & $7-10$ & $8-10$ & $15-17$ & 3 & $\mathrm{Rf}$ \\
\hline
\end{tabular}

A amostra de base do indivíduo Catasetum aff. seccoi $1(4 \mathrm{~mm})$ possui várias de camadas no velame (10), córtex (11) e polos de protoxilema (20). Segundo Moreira \& Isaias (2008) [59], o número de camadas no velame, córtex e polos de protoxilema pode influenciar no diâmetro radicular. No entanto, o indivíduo de Catasetum aff. seccoi 3, mesmo com um número menor de camadas no velame (8), córtex (10) e polos de protoxilema (14), apresentou o mesmo diâmetro que Catasetum aff. seccoi 1. Entretanto, foi observado que em Catasetum aff. seccoi 1 as células 
de velame e córtex são alongadas e volumosas, o que também contribui-o diretamente para que a mesma fosse espessa.

Os dados encontrados nesta pesquisa diferem dos observados por Pedroso-de-Moraes et al. (2012) [21] em um comparativo entre Catasetum discolor e Catasetum pileatum Rchb.f., em que os autores observaram uma relação entre o diâmetro das raízes e o número de polos de protoxilema, onde as que apresentavam maior número de polos eram as mais espessas.

Portanto, concluímos que para as raízes dessas espécies de Catasetum analisadas neste estudo, o volume das células, o número de camadas no velame, córtex e polos de protoxilemas possam estar relacionados ao forófito e a quantidade de nutrientes disponíveis no mesmo. Quanto maior for a disponibilidade dos recursos (água e nutrientes), maior será o tamanho das células e a quantidade de camadas no velame e córtex, assim como o número polos de protoxilemas no indivíduo; estando estes elementos diretamente relacionados ao diâmetro das raízes.

As raízes dos indivíduos aqui analisados compartilham várias características, sendo essa similaridade também observada por Pedroso-de-Moraes (2012) [21] e Silva et al. (2015) [22] tanto em espécies do gênero Catasetum, quanto em outras Catasetinae. Portanto, a similaridade radicular encontrada nas espécies deste estudo corrobora com Chase et al. (2015) [1] na inserção da subtribo Catasetinae na tribo monofilética Cymbidieae.

\section{CONCLUSÃO}

As espécies estudadas compartilham características anatômicas entre si em suas respectivas raízes e folhas. Os dados encontrados estão de acordo com a literatura descrita para o gênero, podendo inferir que esses dados são comuns as espécies de Catasetum. O registro de folhas anfiestomaticas são aqui descritas pela primeira vez para a anatomia do gênero Catasetum. As diferenças encontradas nas raízes relacionadas ao tamanho, volume das células, número de camadas e polos de protoxilema são insuficientes para a diferenciação dessas espécies de Catasetum, incluindo as variações florais de $C$. seccoi. Portanto, torna-se necessária a realização de estudos moleculares que contribuam com a taxonomia e a sistemática dessas espécies de Catasetum, especialmente nas cinco variações morfológicas florais de C. seccoi. Os dados inéditos sobre essas sete espécies de Catasetum e as cinco variantes florais de $C$. aff. seccoi ampliam o conhecimento anatômico para esse gênero e, somados aos estudos anteriores, ajudarão futuras pesquisas sobre informações morfológicas/anatômicas que possam auxiliar na taxonomia e na sistemática da subtribo Catasetinae.

\section{AGRADECIMENTOS}

À fundação de Amparo à Pesquisa e ao Desenvolvimento Cientifico e Tecnológico do Maranhão (FAPEMA) pela concessão da bolsa de estudo ao primeiro autor (processo BM02144/18). A Universidade Estadual do Maranhão (CESC/UEMA) e Universidade Estadual do Piauí (UESPI, Campus Heróis do Jenipapo) pelo apoio logístico e concessão do laboratório para a realização da pesquisa. À Adarilda Petini Benelli e Edlley Max Pessoa pelo fornecimento do material vegetal de duas espécies utilizadas neste estudo. À Josiane Silva Araújo, Ahanna Kamila da Silva, Catarina Silva Lima e Milena Pereira Vilarinho, pelo apoio técnico e o ensino das técnicas histológicas ao primeiro autor.

\section{REFERÊNCIAS BIBLIOGRÁFICAS}

1. Chase MW, Cameron KM, Freudenstein JV, Pridgeon AM, Salazar G, Van Den Berg C, et al. An updated classification of Orchidaceae. Bot J Linnean Soc. 2015 Fev;177(2):151-74. doi: $10.1111 /$ boj. 12234

2. The Brazil Flora Group (BFG). Growing knowledge: an overview of seed plant diversity in Brazil. Rodriguésia. 2015 Out;66(4):1085-113. doi: 10.1590/2175-7860201566411

3. Koch AK, Miranda JC, Hall CF. Flora das Cangas da Serra dos Carajás, Pará, Brasil: Orchidaceae. Rodriguésia. 2018 Jan;69(1):1-24. doi: 10.1590/2175-7860201869115 
4. Pinheiro F, Barros F, Lourenço RA. O que é uma orquídea. In: Barros F, Kerbauy GB, editors. Orquidologia sul-americana. São Paulo: Editora do Instituto de Bot; 2004. p. 11-28.

5. Chase MW, Freudenstein JV, Cameron KM, Barrett RL. DNA data and Orchidaceae systematics: a new phylogenic classification. In: Dixon K, Kell SP, Barrett RL, Cribb PJ, editors. Orchid conservation, Kota Kinabalu: Natural History Publications; 2003. p. 69-89.

6. Pridgeon AM, Cribb PJ, Chase MW, Rasmussen FN, editors. Genera Orchidacearum. Volume 5: Epidendroideae (Part II). Oxford: Oxford University Press Inc.; 2009. 664 p.

7. Batista JAN, Borges KS, Faria MW, Proite K, Ramalho AJ, Salazar GA, et al. Molecular phylogenetics of the species-rich genus Habenaria (Orchidaceae) in the New World based on nuclear and plastid DNA sequences. Mol Phylogen Evol. 2013 Abr;67(1):95-109. doi: 10.1016/j.ympev.2013.01.008

8. Pérez-Escobar OA, Balbuena JA, Gottschling M. Rumbling orchids: How to assess divergent evolution between chloroplast endosymbionts and the nuclear host. Syst Biol. 2016 Set 65(1):51-65. doi: 10.1093/sysbio/syv070

9. Miranda FE, Lacerda KG. Estudos em Catasetinae (Orchidaceae). Bradea. 1992 Nov;6(7):45-60.

10. Romero GA, Carnevali G. Catasetum. In: Pridgeon AM, Cribb PJ, Chase MW, Rasmussen FN, editors. Genera Orchidaceaerum, Epidendroideae-Part II. New York: Oxford University Press; 2009. p. 13-18.

11. Flora do Brasil 2020. Jardim Botânico do Rio de Janeiro [Internet]; 2021 [cited 2021 Jan 29]. Available from: http://floradobrasil.jbrj.gov.br/

12. Hoehne FC. Orchidaceae. In: Hoehne FC. Flora Brasílica. Fasc. 5, Vol. XII: 6. São Paulo: Secretaria da Agricultura, Indústria e Comércio de São Paulo; 1942. 218 p.

13. Benelli AP. Orquídeas de Mato Grosso Genus Catasetum L. C. Rich ex Kunth. 1. ed. Rio de Janeiro: PoD Editora; 2012.

14. Felix LP, Guerra MS. O cariótipo de Nhotoscordum pulchellum (Alliaceae) com ênfase na heterocromatina e nos sítios de rDNA. Bol Soc Argent Bot. 2000 Jan;35(3):283-89.

15. Dressler RL. The orchids: natural history and classification. Cambridge: Harvard University Press; 1981. $250 \mathrm{p}$.

16. Benzing DH. Mineral nutrition. In: Ashton PS, editor. Vascular epiphytes, general biology and related biota. Cambridge (US): University Press Cambridge; 1990. p. 112-51.

17. Franken EP, Pansarin LM, Pansarin E. Osmophore diversity in the Catasetum Cristatum alliance (orchidaceae: Catasetinae). Lankesteriana. 2016 Out;16(3):317-27. doi: 10.15517/lank.v16i3.26649

18. Gomes SM, Somavilla NSDN, Bezerra KMG, Miranda SC, Carvalho PS, Ribeiro DG. Anatomia foliar de espécies de Myrtaceae: contribuições à taxonomia e filogenia. Acta Bot Bras. 2008 Mar;23(1):22338. doi: 10.1590/S0102-33062009000100024

19. Oliveira DM, Silva IV, Rossi AAB. Estudo anatômico foliar de espécies de Citrus com potencial medicinal. Encicl Biosfera. 2014 Jul;10(8):3280-90.

20. Stern WL, Judd WS. Comparative anatomy and systematics of Catasetinae (Orchidaceae). Bot J Linnean Soc. 2001 Jun;136(2):153-78. doi: 10.1111/j.1095-8339.2001.tb00564.x

21. Pedrosa-de-Moraes C, Leal TS, Brescansin RL, Benelli AP, Sajo MG. Radicular anatomy of twelve representatives of the Catasetinae subtribe (Orchidaceae: Cymbidieae). Anais Acad Bras Ciên. 2012 Abr;84(2):455-67. doi: 10.1590/S0001-37652012005000028

22. Silva IV, Oliveira RM, Rossi AAB, Silva AB, Oliveira DM. Use of anatomical root markers for species identification in Catasetum (Orchidaceae) at the Portal da Amazônia region, MT, Brazil. Acta Amazonica. 2015 Mar 451(1):21-8. doi: 10.1590/1809-4392201401832

23. Oliveira MS, Ferreira AWC, Oliveira HC, Pessoa E. Orchids in the central region of eastern Maranhão, Brazil. Rodriguésia. 72:e02582019. 2021. doi: 10.1590/2175-7860202172057

24. Instituto Brasileiro de Geografia e Estatística (IBGE). Cidades [Internet]; 2021 [cited 2021 Jan 29]. Available from: https://cidades.ibge.gov.br

25. Costa CF, Fonseca RS, Almeida DB, Oliveira MS, Oliveira DS, Braga JHP. Espécies utilizadas na arborização em praças do município de Caxias, Maranhão. Soc Bras Arbor Urbana. 2017 Fev;12(1):6578. doi: 10.5380/revsbau.v12i1.63422

26. Oliveira MS, Ferreira AWC, Lopes JRS, Reis JR, Silva Junior WR, Costa JA. Espécies vegetais presentes em praças e avenidas do município de Aldeias Altas, Maranhão, Brasil. Soc Bras Arbor Urbana. 2017 Dez;12(4):13-22. doi: 10.5380/revsbau.v12i4.63585

27. Oliveira MS, Silva EO, Ferreira AWC, Guarçoni EAE. Conhecimento e uso tradicional das espécies madeireiras e medicinais utilizadas no município de Aldeias Altas, Maranhão, Brasil. Encicl Biosfera. 2016 Dez;13(24):1116-73. doi: 10.18677/EnciBio_2016B_109

28. Oliveira MS, Oliveira HC, Ferreira AWC, Silva Junior WR, Silva MJC. Levantamento florístico das espécies utilizadas no paisagismo do município de São João do Sóter, Maranhão, Brasil. Encicl Biosfera. 2019 Jun;16(29): 81-994. doi: 10.18677/EnciBio_2019A80

29. Thiers B [continuously updated] Index herbariorum: a global directory of public herbaria and associated staff. New York Botanical Garden's Virtual Herbarium [Internet]; 2021 [cited 2021 Jan 29]. Available from: http://sweetgum.nybg.org/science/ih/ 
30. Johansen DA. Plant microtechnique. 1st ed. New York: McGraw-Hill Book; 1940. 530 p.

31. Roeser KR. Die Nadel der SchwarzKiefer. Nassen produkt und Kunstwuk der Natur. Mikrokosmos. 1972;61:33-6.

32. Kaiser E. Verfahren zur Herstellung einer tadellosen Glycerin- Gelatine. Botanisch Zentralb; 1880. 180:25-6.

33. Oliveira VC, Sajo MG. Anatomia foliar de espécies epífitas de Orchidaceae. Rev Bras Bot. 1999 Dez;22(3):365-74. doi: 10.1590/S0100-84041999000300003

34. Della-Colleta RCL, Silva IV. Morfoanatomia foliar de microorquídeas de Ornithocephalus Hook. e Psygmorchis Dodson \& Dressler. Acta Bot Bras. 2008 Out;22(4):1068-76. doi: 10.1590/S010233062008000400017

35. Zanenga-Godoy, Costa CG. Anatomia foliar de quatro espécies do gênero Cattleya Lindl. (Orchidaceae) do planalto central brasileiro. Acta Amazonica. 2003;17:101-18.

36. Reis C, Bieras AC, Sajo MG. Anatomia foliar de Melastomataceae do Cerrado do Estado de São Paulo. Rev Bras Bot. 2005 Set;28(3):451-66. doi: 10.1590/S0100-84042005000300004

37. Abbade LC, Paiva PDO, Paiva R, Castro EM, Centofante AR, Oliveira C. Anatomia foliar de ipê-branco (Tabebuia roseo alba (Ridl.) Sand.) - Bignoniaceae, proveniente do cultivo ex vitro e in vitro. Acta Scientiarum Biol Sci. 2009 Jun;31(3):307-11. doi: 10.4025/actascibiolsci.v31i3.1937

38. Rosso SW. The vegetative anatomy of the Cypripedioideae (Orchidaceae). J Linnean Soc (Botany). 1966 Fev;59(379):309-41. doi: doi.org/10.1111/j.1095-8339.1966.tb00066.x

39. Rasmussen H. Orchid stomata - structure, differentiation, function, and phylogeny. In: J. Arditti, editor. Orchid biology: reviews and perspectives, IV. New York (US): Cornell University Press; 1987. p. 10538.

40. Silva IV, Meira RMSA, Azevedo AA, Euclydes RMA. Estratégias anatômicas foliares de treze espécies de Orchidaceae ocorrentes em um campo de altitude no Parque Estadual da Serra do Brigadeiro (PESB) - MG, Brasil. Acta Bot Bras. 2006 Abr;20(3):741-50. doi: 10.1590/S0102-33062006000300023

41. Kurzweil H, Linder HP, Stern WL, Pridgeon AM. Comparative vegetative anatomy and classification of Diseae (Orchidaceae). Bot J Linnean Soc. 1995 Mar; 117(3):171-220.

42. Pridgeon AM. Systematic leaf anatomy of Caladeniinae (Orchidaceae). Bot J Linnean Soc. 1994 Jan;114(1):31-48. doi: 10.1006/boj1.1994.1003

43. Stern WL, Judd WS, Carlsward BS. Systematic and comparative anatomy of Maxillarieae (Orchidaceae), sans Oncidiinae. Bot J Linnean Soc. 2004 Mar;144(3):251-74. doi: 10.1111/j.10958339.2003.00257.x

44. Mauseth JD. Botany: an introduction to plant biology. 2nd ed. London: Saunders College Publishing; 1995. $231 \mathrm{p}$.

45. Bonates LCM. Estudos ecofisiológicos de Orchidaceae da Amazônia II. Anatomia ecológica foliar de espécies com metabolismo CAM de uma campina da Amazônia Central. Acta Amazônica. 1993 Out;23(4):315-48. doi: 10.1590/1809-43921993234348

46. Segecin S, Scatena VL. Morfoanatomia de rizomas e raízes de Tillandsia L. (Bromeliaceae) dos Campos Gerais, PR, Brasil. Acta Bot Bras. 2004 Jun;18(2):253-60. doi: 10.1590/S0102-33062004000200005

47. Proença SL, Sajo MG. Rhizome and root anatomy of 14 species of Bromeliaceae. Rodriguésia. 2008 Mar; 59(1):113-28. doi: 10.1590/2175-7860200859106

48. Silva IV, Scatena VL. Anatomia de raízes de nove espécies de Bromeliaceae (Poales) da região amazônica do estado de Mato Grosso, Brasil. Acta Bot Bras. 2011 Jun;25(3):618-27. doi: 10.1590/S0102-33062011000300015

49. Cortez PA, Silva DC, Alba LFC. Manual prático de morfologia e anatomia vegetal. Ilhéus (BA): Editus; 2016. 92 p.

50. Silva IV, Meira RMSA, Azevedo AA. Anatomia de raízes de espécies de Orchidaceae do Parque Estadual da Serra do Brigadeiro, Minas Gerais. Hoehnea. 2010 Mar;37(1):147-61. doi: 10.1590/S223689062010000100010

51. Pita PB, Menezes NL. Anatomia da raiz de espécies de Dyckia Schult. f. e Encholirium Mart. ex Schult. \& Schult. f. (Bromeliaceae, Pitcairnioideae) da Serra do Cipó (Minas Gerais, Brasil), com especial referência ao velame. Rev Bras Bot. 2002 Mar;25(1):25-34. doi: 10.1590/S0100-84042002000100005

52. Almeida DP, Chaves ALF. Morfo-anatomia de espécie Chrysanthemum leucanthemum (Asteraceae). Biol Ciên Terra. 2001 Mar;1(2):1-7.

53. Sanford WW, Adanlawo I. Velamen and exodermis characters of West African epiphytic orchids in relation to taxonomic grouping and habitat tolerance. Bot J Linnean Soc. 1973 Jun;66(4):307-21. doi: 10.1111/j.1095-8339.1973.tb02178.x

54. Hoehne FC. Iconografia de Orchidaceae do Brasil. São Paulo: Instituto de Botânica; 1949.

55. Pridgeon AM, Stern WL, Benzing DH. Tilosomes in roots of Orchidaceae: Morphology and systematic occurrence. American J Bot. 1983 Mar;70(9):1365-77. doi: 10.2307/2443427

56. Dahlgren RMT, Clifford HT, Yeo PF. The families of the monocotyledons: structure, evolution and taxonomy. Berlin: Springer-Verlag; 1985. 520 p. 
57. Pridgeon AM. The velamen and exodermis of orchid roots. In: Arditti J, editor. Orchid biology: reviews and perspectives. Ithaca (US): Cornell University Press; 1987. p. 30-56.

58. Gonzaga MEB, Gonzaga ALA. Estrutura das orquídeas. Boletim Catarinense de Orquídeas e Bromélias. 1996 Mar;4:2-3.

59. Moreira ASFP, Isaias RMS. Comparative anatomy of the absorption roots of terrestrial and epiphytic orchids. Braz Arch Biol Technol. 2008 Jan;51(1):83-93. 